\title{
Mesure indirecte de la puissance de coupe pour une opération de contournage
}

\author{
Sébastien Auchet ${ }^{1, a}$, Pierre Chevrier ${ }^{1}$, Michel Lacour $^{2}$ Et Paul Lipinski ${ }^{1}$ \\ 1 Laboratoire de Physique et Mécanique des Matériaux, École nationale d’ingénieurs de Metz, CEPGV, Ile du Saulcy, \\ 57045 Metz, France \\ 2 Société de Mécanique Magnétique (S2M), BP 2282, 2 rue des Champs, 27950 Saint Marcel, France
}

Reçu le 14 février 2005, accepté le 13 avril 2005

\begin{abstract}
Résumé - Cette étude a pour objectif de présenter une méthode de mesure indirecte de la puissance de coupe pour une opération de contournage. Nous avons effectué une série d'essais sur une fraiseuse 5 axes Gambin 120CR équipée d'une broche à paliers magnétiques SMB30 développée par la société S2M, broche de $70 \mathrm{~kW}$ de puissance, tournant à $35000 \mathrm{tr} \cdot \mathrm{min}^{-1}$. L'originalité de notre travail porte sur la technique de mesure des efforts de coupe à partir de la mesure des courants de commande des deux paliers magnétiques de la broche. Cette méthode permet d'effectuer des mesures d'efforts au-delà de la plage de bon fonctionnement de la platine Kistler. Ces essais nous ont permis de valider notre méthode de mesure des efforts de coupe pour des paramètres outils variés (rayon de bec, diamètre, nombre de dents) et pour des conditions de coupe différentes (vitesse de coupe, vitesse d'avance, engagement axial et profondeur de passe), lors d'une opération de contournage en fraisage à grande vitesse. Pour chaque essai, nous trouvons un bon accord entre la puissance calculée à partir des mesures des efforts de coupe et la puissance consommée par le moteur de la broche.
\end{abstract}

Mots clés : Efforts de coupe / mesure / paliers magnétiques

Abstract - Indirect measurement of cutting power for peripheral milling operation. The aim of this study is to present an indirect method to measure cutting power for a peripheral milling. Milling tests have been performed on 5-axis Gambin 120CR machine fitted out with the electro-spindle with active magnetic bearings (AMB) SMB30, developped by S2M society. These bearings are not affected by friction and wear. An experimental approach has been developed to determine the cutting power in function of the measured control voltages of the milling spindle's magnetic bearings. The validity of the cutting power calculation method was firstly established for slotting and extend to the case of peripheral milling operation. This indirect method of cutting force determination provides a useful way to estimate tool wear and monitor on-line product quality in high-speed milling.

Key words: Cutting force / measure method / magnetic beargins

\section{Introduction}

En usinage à grande vitesse, la connaissance des efforts de coupe est capitale pour caractériser la qualité de l'usinage et se fait généralement à l'aide des dynamomètres piézoélectriques de la platine Kistler. Pour les vitesses élevées fournies par les nouvelles broches dédiées à l'UGV, cette méthode de mesure ne peut pas être utilisée et il faut créer et développer de nouvelles techniques de mesure permettant d'accéder à ces domaines de fréquences élevées.

\footnotetext{
${ }^{a}$ Auteur pour correspondance:

sebastien.auchet@gmail.com
}

En usinant avec la broche à paliers magnétiques SMB30 développée par S2M, tournant à 35000 tours par minute, soit à une fréquence de rotation de la broche de 583 hertz, nous avons développé une méthode permettant de déterminer les efforts de coupe à partir du traitement des efforts appliqués au rotor par les électroaimants des paliers magnétiques.

Après avoir rappelé le principe et les limites de la mesure d'efforts effectuée à l'aide de la platine Kistler, nous décrivons notre méthode de détermination des efforts de coupe à partir des mesures d'efforts au niveau des paliers magnétiques de la broche. Nous avons effectué une série d'essais pour valider notre méthode, en comparant la puissance de coupe calculée à partir de nos mesures 
d'efforts, à la puissance consommée par le moteur de la broche de fraisage.

\section{Mesure d'efforts en UGV}

La platine Kistler à dynamomètres piézoélectriques est traditionnellement utilisée pour effectuer les mesures d'efforts de coupe. Cependant son utilisation reste peu pratique car il faut fixer les pièces sur la platine, ce qui limite la taille des pièces qu'il est possible d'usiner et oblige à prévoir des points de fixation pour les pièces de dimension acceptable. De plus, le comportement dynamique de la platine et des éléments de bridage de la pièce introduit des distorsions au niveau des signaux mesurés. La méthode de compensation accélérométrique a été utilisée pour améliorer la qualité des mesures d'efforts [1-3]. La première fréquence de résonance des platines Kistler reste faible par rapport aux fréquences d'excitations mesurées en usinage à grande vitesse. Pour une platine Kistler 9255B, par exemple, la plage d'utilisation est limitée à une fréquence d'excitation de $650 \mathrm{~Hz}$ car la première fréquence de résonance est proche de $2 \mathrm{kHz}$. Les fréquences d'excitation des essais réalisés au cours de notre série de mesures se situent entre $730 \mathrm{~Hz}$ et $1500 \mathrm{~Hz}$, ce qui dépasse les capacités de la platine Kistler. Dans ce cas, la compensation accélérométrique ne suffit plus à filtrer les signaux mesurés à l'aide de la platine. C'est pourquoi, nous avons développé une méthode originale, permettant de mesurer les efforts de coupe en utilisant les informations fournies par les paliers magnétiques de la broche d'usinage.

\section{La broche de fraisage à grande vitesse à paliers magnétiques actifs}

\subsection{Principe de fonctionnement}

Le palier magnétique est composé de deux paires d'électroaimants qui appliquent des efforts uniaxiaux au rotor. L'effort, proportionnel aux tensions de commande des bobines électriques, peut être modulé. C'est cette particularité des paliers magnétiques qui, en réagissant immédiatement à toutes les modifications de chargement du rotor, comme par exemple les efforts intermittents de coupe en bout d'outil ou le balourd du rotor, est utilisée pour maintenir l'axe de rotation de l'arbre dans sa position centrale.

Le déplacement du rotor est mesuré par des capteurs de position. Lorsqu'une erreur de position est détectée par les capteurs, la boucle de contrôle module le courant dans chaque bobine pour faire varier l'effort appliqué par les électroaimants sur le rotor afin de le replacer dans sa position de repos (Fig. 1).

\subsection{Description de la broche}

La broche SMB30 peut développer jusqu'à $70 \mathrm{~kW}$ de puissance en tournant à 35000 tr.min ${ }^{-1}$. Elle se compose de deux paliers magnétiques actifs radiaux (PMA 1 et PMA 2) et d'un palier magnétique axial (PMA 3).

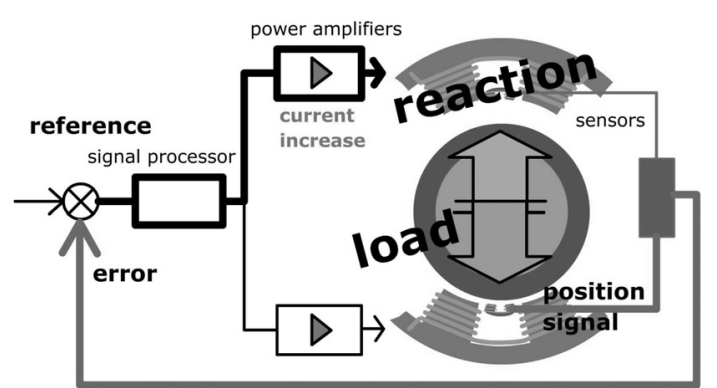

Fig. 1. Boucle de rétroaction d'un palier magnétique actif.

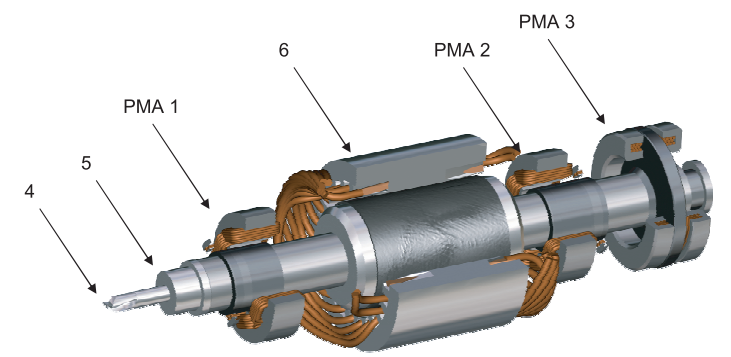

Fig. 2. Schéma de la broche d'usinage à grande vitesse développée par S2M.

L'outil (Réf. 4) est fretté dans le porte outil qui est maintenu au rotor par le serreur d'outil OTT (Réf. 5). Le moteur entraînant la broche en rotation est situé entre les deux PMA axiaux (Réf. 6).

\subsection{Méthode de mesure indirecte des efforts de coupe}

Nous avons développé une méthode permettant de déterminer les efforts de coupe à partir du traitement des efforts appliqués au rotor par les électroaimants des paliers magnétiques actifs radiaux (PMA) de la broche. Les efforts de coupe sont déterminés à partir des efforts mesurés au niveau des paliers magnétiques en utilisant des fonctions de transfert entre les paliers magnétiques de la broche et le bout de l'outil. Les fonctions de transfert utilisées sont mesurées lorsque la broche n'est pas en rotation. Ces fonctions sont également valables lorsque la broche est en rotation en dehors des fréquences proches des modes de flexion de l'ensemble rotor/porteoutil/outil. Au voisinage de ces fréquences, les effets gyroscopiques sont à l'origine du phénomène de dédoublement des modes, ce qui modifie la fonction de transfert. Ainsi, notre méthode de mesure des efforts de coupe n'est valable que si aucune fréquence n'excite les modes de flexion du rotor au cours de l'usinage. En particulier, elle ne peut donc pas être utilisée lors de l'apparition du phénomène de broutage régénératif.

\section{Description de la campagne d'essais}

\subsection{Les paramètres étudiés}

Nos essais doivent permettre de tester si la méthode de mesure des efforts de coupe est valable pour des 
paramètres outils variés (rayon de bec, diamètre, nombre de dents) et des conditions de coupe différentes (vitesse de coupe, vitesse d'avance, engagement axial et profondeur de passe), lors d'une opération de contournage en concordance. Les usinages ont été réalisés sur une fraiseuse 5 axes Gambin 120CR équipée d'une broche à paliers magnétiques SMB30 développée par la société S2M. Chaque essai de contournage a été réalisé avec des outils à plaquettes de la marque CERATIZIT. Les caractéristiques de ces outils sont présentées dans le tableau 1.

Les valeurs des différents paramètres outil et les différents paramètres de coupe sont présentées dans le tableau 2.

Nous avons veillé à ce que le montage de buses de microlubrification rigides permette de maintenir le paramètre lubrification identique pour chaque essai. Les deux engagements choisis de $9 \mathrm{~mm}$ et $27 \mathrm{~mm}$ correspondent respectivement à, environ, un quart et trois quarts du diamètre de l'outil en prise avec la matière. Les usinages sont effectués dans de l'AU4G.

\subsection{Les grandeurs étudiées}

Pour vérifier la qualité de l'usinage, nous avons mesuré la rugosité en fond et en flanc avec le profilomètre Mahr de modèle « Perthomètre BK ». Nous avons ensuite comparé la puissance spécifique de coupe mesurée à partir des efforts de coupe, à la puissance consommée par le moteur. Nous cherchons par ce biais à valider la mesure des efforts de coupe dans le domaine de fréquence compris entre $730 \mathrm{~Hz}$ et $1500 \mathrm{~Hz}$, ce qui est l'objectif principal de notre étude. La puissance consommée par le moteur comprend la puissance de coupe mais également la puissance dissipée par les frottements hydrodynamiques. Pour déterminer la puissance de coupe, nous avons tout d'abord déterminé les efforts en bout d'outil $F_{x}$ et $F_{y}$ à partir de la mesure des courants de commande des paliers magnétiques de la broche. Pour une rotation $\theta$ de l'outil par rapport à la direction d'avance, l'effort tangentiel instantané de coupe $F_{\mathrm{T}}$ en concordance est :

$$
F_{\mathrm{T}}=\cos (\theta) F_{x}-\sin (\theta) F_{y}
$$

Le couple moyen pour une fraise à $N$ dents s'écrit [4] :

$$
C=\frac{N}{2 \pi} \int_{0}^{\theta \mathrm{im}} F_{\mathrm{T}} R \mathrm{~d} \beta
$$

avec $: R$, le rayon de l'outil, $\theta^{\text {im }}$ l'angle d'immersion.

La puissance moyenne de coupe pour une fréquence de rotation de la broche $\omega$ est :

$$
P=C \omega
$$

\subsection{Essais réalisés}

Les douze configurations de paramètres choisis sont présentées dans le tableau 3.

Afin de consolider les résultats, trois usinages sont réalisés pour chaque configuration.
Tableau 1. Caractéristiques des outils et des plaquettes utilisées lors des essais.

\begin{tabular}{|c|c|c|c|}
\hline Référence outil & $\begin{array}{c}\text { Nombre } \\
\text { dents }\end{array}$ & $\begin{array}{c}\text { Diamètre } \\
(\mathrm{mm})\end{array}$ & $\begin{array}{c}\text { Longeur aur-delà du } \\
\text { cône HSK }(\mathrm{mm})\end{array}$ \\
\hline MHPC 32 R 02 - 19 & 2 & 32 & 75 \\
\hline MHPC 32 R 03 - 19 & 3 & 32 & 75 \\
\hline MHPC 40 R 02 - 19 & 2 & 32 & 75 \\
\hline MHPC 40 R 03 - 19 & 3 & 32 & 75 \\
\hline Référence plaquettes & $\begin{array}{c}\text { Longueur } \\
(\mathrm{mm})\end{array}$ & $\begin{array}{c}\text { Épaisseur } \\
(\mathrm{mm})\end{array}$ & Rayon de bec $(\mathrm{mm})$ \\
\hline $\begin{array}{c}\text { XDHX 190420FR -27P } \\
\text { HW K15 }\end{array}$ & 19 & 4 & 2 \\
\hline $\begin{array}{c}\text { XDHX 190404FR -27P } \\
\text { HW K15 }\end{array}$ & 19 & 4 & 0,4 \\
\hline
\end{tabular}

\begin{tabular}{|c|c|c|c|c|}
\hline $\begin{array}{c}\text { Rayon de bec } \\
(\mathrm{mm})\end{array}$ & \multicolumn{2}{|c|}{0,4} & \multicolumn{2}{|c|}{2} \\
\hline $\begin{array}{c}\text { Diamètre } \\
(\mathrm{mm})\end{array}$ & \multicolumn{2}{|c|}{32} & \multicolumn{2}{|c|}{40} \\
\hline Nombre dents & \multicolumn{2}{|c|}{2} & \multicolumn{2}{|c|}{3} \\
\hline$V_{c}\left(m \cdot m^{-1}\right)$ & \multicolumn{2}{|c|}{2199} & \multicolumn{2}{|c|}{3016} \\
\hline$F_{Z}\left(m m \cdot\right.$ dent $\left.^{-1}\right)$ & \multicolumn{2}{|c|}{0,07} & \multicolumn{2}{|c|}{0,18} \\
\hline$A_{\mathrm{e}}(\mathrm{mm})$ & \multicolumn{2}{|c|}{9} & \multicolumn{2}{|c|}{27} \\
\hline$A_{p}(\mathrm{~mm})$ & 3 & 5 & 7 & 9 \\
\hline
\end{tabular}

Tableau 2. Valeurs des différents paramètres étudiés lors de la série d'essai.

\section{Résultats - discussion}

\section{1 Étude de la rugosité moyenne de fond et de flanc}

Les valeurs de la rugosité en fond et en flanc sont représentées dans le tableau 4. Les faibles valeurs de $R a$ montrent la qualité de l'état de surface de nos usinages. On peut aussi constater que, pour chaque configuration, les résultats du $R a$ de flanc et du $R a$ de fond des trois essais sont très proches, ce qui montre la cohérence et la reproductibilité de nos résultats.

\subsection{Comparaison entre la puissance consommée par le moteur et la puissance de coupe}

Les efforts de coupe des configurations d'essai 5 et 6 n'ont pas pu être déterminées par notre méthode de mesure décrite à la section 2.3, car le premier mode de flexion de l'ensemble rotor/porte-rotor/outil a été excité pour ces usinages.

Lorsque la broche, en rotation à 30000 tr.min $^{-1}$, n'usine pas, la puissance consommée par le moteur de la broche est comprise entre 1 et $2 \mathrm{~kW}$. Cette puissance est consommée par les pertes dues aux frottements aérodynamiques, ce qui correspond à la différence 
Tableau 3. Essais réalisés.

\begin{tabular}{|c|c|c|c|c|c|c|c|c|}
\hline $\begin{array}{c}\text { No. } \\
\text { essai }\end{array}$ & $\begin{array}{l}\text { Rayon de } \\
\text { bec (mm) }\end{array}$ & $A_{\mathrm{p}}(\mathrm{mm})$ & $V_{c}\left(\operatorname{m} \cdot \min ^{-1}\right)$ & $\begin{array}{c}F_{Z} \\
\left(\text { mm.dent }^{-1}\right)\end{array}$ & $A_{\mathrm{e}}(\mathrm{mm})$ & $\begin{array}{c}\text { Nombre } \\
\text { dents }\end{array}$ & $\begin{array}{l}\text { Diamètre } \\
(\mathrm{mm})\end{array}$ & $N\left(\right.$ tr. $\left.\min ^{-1}\right)$ \\
\hline 1 & 0,4 & 3 & 2199 & 0,07 & 9 & 2 & 32 & 21,875 \\
\hline 2 & 0,4 & 3 & 3016 & 0,18 & 27 & 3 & 40 & 30,000 \\
\hline 3 & 0,4 & 5 & 2199 & 0,07 & 9 & 2 & 40 & 21,875 \\
\hline 4 & 0,4 & 5 & 3016 & 0,18 & 27 & 3 & 32 & 30,000 \\
\hline 5 & 0,4 & 7 & 2199 & 0,07 & 27 & 3 & 32 & 21,875 \\
\hline 6 & 0,4 & 9 & 2199 & 0,07 & 27 & 3 & 40 & 21,875 \\
\hline 7 & 2 & 3 & 3016 & 0,07 & 27 & 2 & 40 & 30,000 \\
\hline 8 & 2 & 5 & 3016 & 0,07 & 27 & 2 & 32 & 30,000 \\
\hline 9 & 2 & 7 & 2199 & 0,18 & 27 & 2 & 32 & 21,875 \\
\hline 10 & 2 & 7 & 3016 & 0,07 & 9 & 3 & 40 & 30,000 \\
\hline 11 & 2 & 9 & 2199 & 0,18 & 27 & 2 & 40 & 21,875 \\
\hline 12 & 2 & 9 & 3016 & 0,07 & 9 & 3 & 32 & 30,000 \\
\hline
\end{tabular}

Tableau 4. Mesures de rugosité.

\begin{tabular}{|c|c|c|c|c|c|c|}
\cline { 2 - 7 } \multicolumn{1}{c|}{} & \multicolumn{3}{c|}{$R a$ flanc } & \multicolumn{3}{c|}{$R a$ fond } \\
\hline No.essai & Usinage 1 & Usinage 2 & Usinage 3 & Usinage 1 & Usinage 2 & Usinage 3 \\
\hline 1 & 0,15 & 0,17 & 0,13 & 0,16 & 0,17 & 0,16 \\
\hline 2 & 0,35 & 0,41 & 0,37 & 0,84 & 0,95 & 0,77 \\
\hline 3 & 0,14 & 0,18 & 0,18 & 0,15 & 0,16 & 0,12 \\
\hline 4 & 1,05 & 0,91 & 0,98 & 0,65 & 0,53 & 0,65 \\
\hline 5 & 0,71 & 0,58 & 0,82 & 0,42 & 0,43 & 0,56 \\
\hline 6 & 0,53 & 0,57 & 0,71 & 0,4 & 0,52 & 0,57 \\
\hline 7 & 0,13 & 0,11 & 0,15 & 0,22 & 0,42 & 0,29 \\
\hline 8 & 0,67 & 0,58 & 0,66 & 0,47 & 0,29 & 0,38 \\
\hline 9 & 0,35 & 0,39 & 0,33 & 0,47 & 0,39 & 0,55 \\
\hline 10 & 0,14 & 0,12 & 0,12 & 0,34 & 0,25 & 0,34 \\
\hline 11 & 0,25 & 0,21 & 0,27 & 0,59 & 0,88 & 0,8 \\
\hline 12 & 0,25 & 0,2 & 0,15 & 0,26 & 0,25 & 0,31 \\
\hline
\end{tabular}

Tableau 5. Comparaison entre la puissance calculée à partir des efforts de coupe et la puissance consommée par le moteur.

\begin{tabular}{|c|c|c|c|c|c|c|}
\cline { 2 - 7 } \multicolumn{1}{c|}{} & \multicolumn{3}{c|}{$\begin{array}{c}\text { Puissance calculée à partir des } \\
\text { efforts de coupe (en watts) }\end{array}$} & \multicolumn{3}{c|}{$\begin{array}{c}\text { Puissance consommée par le } \\
\text { moteur (en watts) }\end{array}$} \\
\hline $\mathrm{N}^{\circ}$ & Usinage 1 & Usinage 2 & Usinage 3 & Usinage 1 & Usinage 2 & Usinage 3 \\
\hline 1 & 1404 & 1542 & 1819 & 1727 & 1727 & 1727 \\
\hline 2 & 20231 & 19878 & 20430 & 21714 & 21720 & 20800 \\
\hline 3 & 1575 & 1883 & 1905 & 1727 & 1727 & 2015 \\
\hline 4 & 32122 & 31270 & 32309 & 35532 & 33558 & 35532 \\
\hline 7 & 7078 & 7447 & 7192 & 6316 & 5132 & 6317 \\
\hline 8 & 7308 & 7325 & 8019 & 9870 & 9080 & 9430 \\
\hline 9 & 23797 & 23718 & 23552 & 24326 & 24469 & 24181 \\
\hline 10 & 7070 & 7708 & 6950 & 7896 & 6317 & 7106 \\
\hline 11 & 30806 & 31654 & 30155 & 32136 & 32242 & 33129 \\
\hline 12 & 8100 & 9048 & 8302 & 7896 & 9080 & 9475 \\
\hline
\end{tabular}

constatée entre les deux puissances comparées dans le tableau 5. Dans ces conditions, nous pouvons voir sur le tableau 5, l'excellent accord entre les valeurs de la puissance que nous avons calculée à partir des efforts de coupe et la puissance consommée par le moteur.

La comparaison entre la puissance consommée par le moteur et la puissance de coupe permet de valider notre méthode de mesure de l'effort de coupe dans un domaine de fréquence qui ne permet pas une comparaison avec une platine Kistler.

\subsection{Conclusion}

Dans ce travail, nous avons mis au point une méthode originale de calcul des efforts de coupe à partir de la mesure des forces appliquées au rotor par les électroaimants des PMA de la broche. Les efforts de coupe sont déterminés à partir des efforts mesurés au niveau des paliers magnétiques en utilisant des fonctions de transfert entre les paliers magnétiques de la broche et le bout de l'outil.

Les fréquences d'excitation des usinages réalisés au cours de notre série d'essais, entre $730 \mathrm{~Hz}$ et $1500 \mathrm{~Hz}$, se situent au-delà de la bande de fréquence de fonctionnement de la platine Kistler. Nous avons validé notre méthode de mesure des efforts dans ce domaine de fréquence en comparant la puissance de coupe calculée à partir de la mesure des efforts de coupe à la puissance consommée par le moteur.

Nous avons montré qu'actuellement notre méthode de mesure n'est valable que si aucune fréquence n'excite les modes de flexion du rotor au cours de l'usinage. La prise en compte du dédoublement de mode induit par les effets gyroscopiques dans les fonctions de transfert entre les efforts au niveau des paliers et les efforts de coupe devrait permettre d'étendre notre méthode de mesure aux usinages présentant le phénomène de broutage régénératif.

\section{Références}

[1] N. Tounsi, A. Otho, Dynamic cutting force measuring, Machine Tools \& Manufacture, $\mathrm{N}^{\circ}$ 1157-1170, 2000

[2] F. Lapujoulade, Measuring of cutting forces during fast transient periods, First French and German Conference on High Speed Machining, Metz, June 1997, pp. $372-376$

[3] F. Lapujoulade, G. Coffignal, J. Pimont, Évaluation des forces de coupe en fraisage à grande vitesse, $2^{\mathrm{e}}$ conférence internationale IDMME'98, UTC Compiègne, mai 1998

[4] Y. Altintas, Manufacturing Automation, Cambridge University Press, ISBN 0-521-65029-1, 2000, pp. 37-38 